\title{
The processing of hierarchical stimuli: Effects of retinal locus, locational uncertainty, and stimulus identity
}

\author{
MARVIN R. LAMB and LYNN C. ROBERTSON \\ Veterans Administration Medical Center, Martinez, California \\ and University of California, School of Medicine, Davis, California
}

\begin{abstract}
Three experiments examined the effects of changes in retinal locus and locational uncertainty in the processing of hierarchical stimuli. In Experiment 1, stimuli were presented randomly in the left, center, or right portions of a display. Central presentation decreased reaction times for identifying small letters presented within a hierarchical stimulus pattern (i.e., local letters) but not for a single small letter presented alone. In Experiment 2, all stimuli were presented centrally, thus eliminating the locational uncertainty that existed in Experiment 1. The elimination of locational uncertainty resulted in faster reaction times (as compared with the central data of Experiment 1) for identifying small letters, whether or not they appeared in a hierarchical pattern. In Experiment 3, eye movements were monitored and eliminated as a possible source of these effects. The results are discussed in terms of possible effects of an attentional "spotlight" on hierarchical stimulus processing. It was also found that the identity of the target letter (i.e., whether it was an $\mathrm{H}$ or an S) had a large effect on performance. Finally, in contrast to earlier findings (Hoffman, 1980; Martin, 1979), the response-time advantage at a given level and the amount of Stroop-type interference produced at the other level did not always covary, suggesting that these two effects may reflect the operation of separate mechanisms.
\end{abstract}

The relationship between the perception of parts and wholes has long been a central issue in the study of visual perception (Koffka, 1935; Titchner, 1909). One view is that perception of the overall scene is built up from an analysis of its parts. The opposing view is that the whole is perceived first and only later is information about the parts extracted (see Robertson, 1986, for an elaboration). The extreme versions of these two views predict a strict order of processing. In one, the whole cannot be perceived until the parts have been analyzed. In the other, the parts cannot be perceived until the whole has been analyzed.

Navon (1977) attempted to test these hypotheses directly. He presented hierarchical stimuli similar to those shown in Figure 1, and compared the time taken to identify the local versus the global letters. Navon found that reaction times (RTs) were faster for global than for local letters. In addition, RTs for local letters were significantly slowed when the global letter conflicted with the local letter (e.g., local Ss forming a global $\mathrm{H}$ ) as opposed to when the global letter was consistent with the local letter (e.g., local Ss forming a global S). In contrast, RT to global letters was unaffected by the identity of the local letter. This unidirectional Stroop (1935) type of interference suggests that global information was available at the time of

This research was supported by the Medical Research Council of the Veterans Administration and NIAAA Grant AA06637 awarded to the second author. Correspondence concerning this article should be sent to Marvin R. Lamb, Research 151, Veterans Administration Medical Center, 150 Muir Road, Martinez, CA 94553.
CONSISTENT INCONSISTENT SINGLE

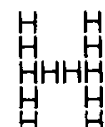

5

H

5

$\begin{array}{llll}5555 & H H H H & 8 & 8 \\ 5555 & H H H H & 8000 \\ 5555 & H H H H & 8008 & 8008 \\ & H & 8 & 0008\end{array}$

Figure 1. Drawings of the stimulus set used in the present experiments. Drawings are to scale, but are smaller than the actual stimuli.

local processing but not vice versa. These findings led Navon to suggest that visual perception proceeds in a global-to-local direction, the so-called global precedence hypothesis.

Subsequent research has shown that these effects are not universal (Grice, Canham, \& Boroughs, 1983; Hoffman, 1980; Kimchi \& Palmer, 1982; Kinchla \& Wolfe, 1979; Martin, 1979; Pomerantz, 1983; Ward, 1982). For example, Kinchla and Wolfe (1979) found that the relative speed with which the local and global levels were processed depended on the visual angle of the stimulus pattern. Global forms were identified faster when the stimulus display subtended less than about $7^{\circ}$ visual angle, but local forms were identified faster with larger displays. 
Martin (1979) found a global advantage when a relatively large number of local elements made up the global form, but a local advantage when fewer local elements were used. Furthermore, Martin found greater interference on local processing when global targets were identified faster, findings parallel to those of Navon (1977), but greater interference on global processing when local targets were identified faster. Hoffman (1980) showed that the relative speed with which local and global targets were identified could be reversed by degrading the information at the local or global levels. Like Martin (1979), Hoffman found greater interference for whichever level was processed more slowly.

Other investigators (Boer \& Keuss, 1982; Miller, 1981) have shown that local-level information is processed before decisions about the global level are made. For example, Miller had subjects search for target letters that could appear at the local level, the global level, or both levels on any given trial. He found that subjects were faster at responding to targets at the global than at the local level, but also that they were faster still when a target appeared at both levels. Thus, the addition of a target at the local level improved performance relative to the global-only condition. This could occur only if local-level information was available at the time global decisions were made.

Findings such as these seem to eliminate a strong version of the global-precedence hypothesis, one that posits a strictly sequential global-to-local order of processing. As a result, several investigators (Boer \& Keuss, 1982; Miller, 1981; Navon, 1981) have agreed that global and local processing can occur in parallel or at least proceed with a similar time course.

It has been suggested that findings such as those described so far are best explained simply by referring to the relative discriminability of the stimuli at the local and global levels (Grice et al,, 1983; Pomerantz, 1983). For example, Pomerantz (1983), presented hierarchical stimuli $3^{\circ}$ out from center. Close inspection of the data reveals that with this type of presentation, Pomerantz replicated the findings of Navon (1977), who also used peripheral presentation. That is, global targets were identified faster and Stroop-type interference occurred only at the local level. In a second experiment, Pomerantz presented the stimuli centrally, arguing that foveal presentation should produce a relatively larger increase in discriminability at the local level than at the global level. With central presentation, local targets were identified as quickly as global targets and Stroop-type interference was symmetric.

Pomerantz (1983) argued that local processing was facilitated for stimuli presented centrally (Experiment 2) compared with those presented peripherally (Experiment 1) because of the increased acuity that accompanies foveal presentation. It cannot be denied that acuity improves with foveal presentation, and it is reasonable to expect such an improvement to affect speed of processing. However, it is also true that stimuli occurred randomly in one of four locations on any given trial in his first experiment but always in the same location in his second experiment. This difference in locational uncertainty may have resulted in improved performance for reasons other than changes in acuity. For example, subjects had to monitor a larger area of space for peripherally as opposed to centrally presented stimuli, and there is ample evidence that (1) the area encompassed by an attentional "spotlight" varies according to task demands, and (2) processing efficiency within the attended area increases as the size of the spotlight decreases (Eriksen \& St. James, 1986; Eriksen \& Yeh, 1985; Johnston \& Dark, 1986; Jonides, 1983). Thus it is possible that at least some of the improvement in performance observed by Pomerantz for centrally presented stimuli may be attributable to the operation of a smaller attentional spotlight in his Experiment 2.

\section{EXPERIMENT 1}

The purpose of the first experiment was to examine hierarchical stimulus processing in a situation where central and peripheral stimulus presentations would differ in retinal locus but would not differ in locational uncertainty. Locational uncertainty was equated for central and peripheral presentations by making the location at which stimuli appeared on any given trial uncertain for both types of presentation. Subjects viewed the stimuli shown in Figure 1 presented randomly on the left, center, or right portion of a video display. Thus, the location at which stimuli occurred was uncertain, but equally so for all three positions. In terms of the attentional hypothesis proposed above, since the location at which the stimulus appeared on any given trial could not be predicted, the attended area should be large enough to include all three potential locations. As a result, stimuli presented centrally should be processed in the context of the same large attentional spotlight as those presented peripherally. However, any difference in acuity resulting from central versus peripheral presentation would remain with the current procedure. Thus, any difference in performance between central and peripheral presentation in the present experiment could be attributed to differences in acuity at the two locations but not to differences in locational uncertainty.

\section{Method}

Subjects. Sixteen right-handed paid volunteers served as subjects. They ranged in age from 23 to 41 years $(M=32.1, S D=5.6)$. All subjects had normal or corrected-to-normal vision.

Apparatus and Stimuli. The stimuli were generated on a Princeton Graphics SR-12 monitor controlled by an IBM PC-XT computer with Sigma Designs Graphic Dazzler I and Enhancer cards. All stimulus events were white on a dark surround. Stimulus timing (onset, offset, and duration) was tied to the vertical sync pulse. All other events (responses, ITI, etc.) were timed using the $\mathbf{8 2 5 3}$ chip set to a 1-msec base. The status of the response keys was monitored via the game port.

A small square presented in the center of the monitor served as the fixation point. It subtended approximately $.21^{\circ}$ visual angle. A set of hierarchical stimuli formed from the letters $H$ and $S$ were also used (see Figure 1). Large letters were constructed from the 
appropriate placement of small letters in a 4 (horizontal) $\times 5$ (vertical) matrix. Small letters subtended approximately $.64^{\circ}$ visual angle vertically and $.42^{\circ}$ horizontally. Large letters subtended about $3.6^{\circ}$ visual angle vertically and $2.3^{\circ}$ horizontally.

The letters $\mathbf{H}$ and $\mathbf{S}$ served as targets. In the Consistent condition, the same letter appeared at the local and global levels (i.e., both local and global letters were $H$ or both were S). In the Inconsistent condition, the local and global letters were different (i.e., global $\mathbf{H}$ and local S or global S and local $\mathbf{H}$ ). In the Single condition, the stimulus was either (1) one small letter presented alone or (2) a large letter composed of small Os, as shown in Figure 1.

The Single condition was included in the present experiment as a control for the possible effects of size. That is, performance might differ in the locally and globally directed conditions because letters at the local level are smaller than letters at the global level rather than because of their relative positions in the hierarchy. The choice of an appropriate size control is somewhat problematic, however. Navon (1977) used a local control stimulus in which small target letters (H or S) made up a "neutral" letter (an $O$ ). These control more for response competition than for size, but they do have the advantage of holding constant possible effects from such things as lateral masking. On the other hand, one problem with them is that small letters in the configuration of an $O$ may still be viewed by the subject as the local level of a hierarchical stimulus. If the processing of small letters differs depending on whether or not they are part of a hierarchical stimulus, then this control would not be appropriate. To avoid this problem, we used a single small letter as our local control stimulus. As will be reported below, the differences between a single small target and local targets in a nontarget O seem not to be crucial, since our results replicate those of Navon's neutral condition.

A masking stimulus was used which consisted of a grid whose overall horizontal and vertical dimensions were identical to those of the hierarchical stimulus patterns. There were 7 vertical and 10 horizontal parallel lines in the grid, and all lines were separated by $.42^{\circ}$ visual angle.

Procedure. A subject sat with his/her head approximately $54 \mathrm{~cm}$ from the front of the CRT screen. A 500-msec tone announced the beginning of each trial. The tone was followed, after $100 \mathrm{msec}$, by a 500 -msec presentation of the central fixation point. The subjects were instructed to look directly at the fixation point and not to move their eyes. The fixation point was followed immediately by a $100-$ msec presentation of one of the stimulus patterns. The stimulus pattern appeared randomly and equally often in the left, center, or right portion of the screen. Peripheral stimuli appeared $2.7^{\circ}$ of visual angle out from the fixation point, measured to the inner edge of the stimulus pattern. The stimulus was replaced by a mask that occurred in the same location as the stimulus pattern on that trial. The mask remained on until the subject responded or until the 3,000-msec interval allowed for a response had elapsed. There was a 1,000 -msec intertrial interval. These procedures were adopted to approximate those of Navon (1977) as closely as possible.

Stimuli were presented in four blocks of trials, 324 trials in all. Each block began with 9 warm-up trials which were not included in the analysis. In the locally directed condition, the small letters served as targets and subjects indicated the identity of the target (H or S) by pressing one of two keys. In the globally directed condition, the large letter served as the target. Half of the subjects were directed globally for Blocks 1 and 2 and locally for Blocks 3 and 4. For the other half, the order was reversed. There were 99 trials in Blocks 1 and 3 and 63 trials in Blocks 2 and 4. The unequal block sizes were chosen solely to equate the present paradigm with other ongoing experiments in our laboratory. Before data collection began, the subjects received a block of 18 globally directed practice trials and a block of 18 locally directed practice trials. The subjects were directed to the relevant level before the start of each block, both before practice and data collection. The order in which the globally/locally directed conditions were presented was the same for the practice and data trials for any given subject. Target letter ( $\mathrm{H}$ and S), consistency condition (Consistent, Inconsistent, and Single), and location (left, center, and right) were completely counterbalanced within each block. Stimuli were presented randomly with the restrictions that the target could not be the same letter or appear in the same location on more than four consecutive trials.

Half of the subjects used the right hand to respond, and half used the left. Subjects using the right hand pressed the $H$ key with the index finger and the $S$ key with the middle finger. Subjects usin the left hand used the opposite fingers for the $\mathrm{H}$ and $\mathrm{S}$ responses. The order in which the globally/locally directed conditions were presented and hand of responding were counterbalanced between subjects. All subjects were instructed to respond as quickly as possible while keeping errors to a minimum. RT (measured from stimulus pattern onset to key closure) and errors were recorded.

\section{Results and Discussion}

The error rate was low $(M=4.5 \%)$, and when RT and errors were compared there was no indication of a speedaccuracy tradeoff $(r=+.20)$. The error data were not analyzed further. The RT data were analyzed using a repeated measures analysis of variance (ANOVA) with level (local vs. global), consistency (consistent, single, or inconsistent), location (left, center, or right), and target letter (H or $\mathbf{S})$ as factors. Median RTs were calculated for each cell in the design, and the data reported in the figures and in the ANOVAs are means of those medians. The same procedure was followed in analyzing the RT data of Experiments 2 and 3.

Overall, the pattern of results was quite similar to that found by Navon (1977, Experiment 3). First, RTs were faster when subjects were directed globally than when they were directed locally $[F(1,15)=11.69, p<.01]$. There was also a large effect of consistency $[F(2,30)=60.54$, $p<.001]$, RTs being slower in the Inconsistent than in the Consistent $[F(1,15)=113.29, p<.001]$ or Single $[F(1,15)=62.86, p<.001]$ conditions. In addition, the effect of consistency was greater when subjects were directed locally than when they were directed globally, as evidenced by a significant level $\times$ consistency interaction $[F(2,30)=15.44, p<.001]$. There was also no difference in the global advantage between Consistent and Single conditions (a finding also consistent with Navon's, despite the difference in the type of control stimuli used).

While the overall pattern of results in the present experiment resembled those found by Navon (1977), the pattern differed for stimuli presented at different locations on the screen (see Figure 2) in a manner similar to that observed by Pomerantz (1983). The main effect of location was significant $[F(2,30)=11.67, p<.001]$, reflecting the fact that responses were faster for stimuli presented centrally than for those presented to the left $[F(1,15)=$ $16.56, p<.001]$ or to the right $[F(1,15)=17.17$, $p<.001]$. Location also interacted with level $[F(2,30)$ $=25.26, p<.001]$, reflecting the fact that the significant global advantage observed in the periphery $[F(1,15)$ $=20.29, p<.001$, for the left and $F(1,15)=17.46$, $p<.001$, for the right] was not observed for stimuli presented centrally. The effect of consistency was also 
larger in the periphery than in the center, as demonstrated by a significant location $x$ consistency interaction $[F(4,60)=3.40, p<.05]$. Finally, the level $\times$ consistency interaction that served as the basis for Navon's global-precedence hypothesis was much more pronounced for stimuli presented peripherally than for stimuli presented centrally, as evidenced by a significant location $\times$ level $\times$ consistency interaction $[F(4,60)=12.08$, $p<.001]$.

The effects of location observed in the present experiment were quite similar to those found by Pomerantz (1983, Experiments 1 and 2). Pomerantz attributed the difference he observed between central and peripheral presentation to a difference in acuity at the two locations. Another possible explanation for location differences could be a difference in the degree of lateral masking. It is well known that lateral masking can have greater effects in the periphery than centrally, and it is possible that this contributed to the global RT advantage in the periphery.

There are some findings in the present data that are consistent with this interpretation and others that are not. The consistency $\times$ location $\times$ level interaction reported above was due in large part to the fact that performance improved in the locally directed condition when stimuli were presented centrally, but only in the Consistent and Inconsistent conditions. There was no indication of a change in performance as a function of location for the Single condition (see Figure 2). That is, RTs for the locally directed condition were shorter with central than with

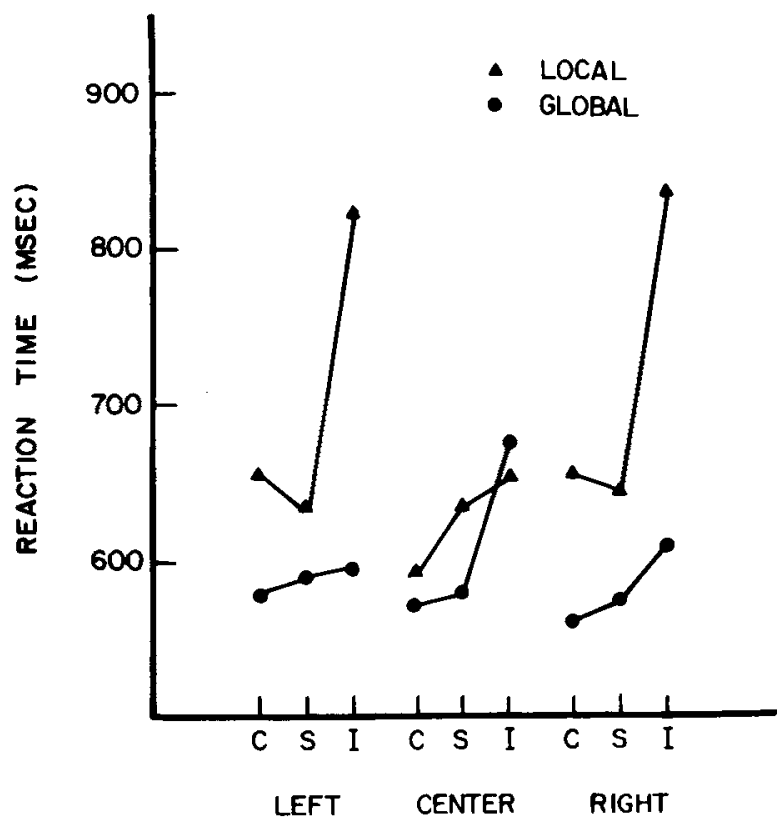

Figure 2. Reaction times for Experiment 1 for locally (triangles) and globally (circles) directed trials as a function of consistency, for stimuli presented on the left, center, or right portions of the display. (C = Consistent condition, $\mathrm{S}=$ Single condition, $\mathrm{I}=$ Inconsistent condition.) peripheral presentation in the Consistent condition $[F(1,15)=10.60, p<.01$, for the left and $F(1,15)=$ $14.63, p<.01$, for the right] and in the Inconsistent condition $[F(1,15)=22.58, p<.001$, for the left and $F(1,15)=28.13, p<.001$, for the right], but not in the Single condition. Thus, peripheral presentation decreased the speed with which "small" letters could be identified, but only when those letters occurred within a hierarchical stimulus. However, if the difference between global and local functions could be attributed to lateral masking, there should have been no difference between the two single stimuli where lateral masking would not occur. Yet, Figure 2 shows that the difference is about the same for the single condition and the consistent condition where lateral masking would have occurred. Lateral masking is further questioned by the fact that the differences between the global and local RTs over Consistent, Single, and Inconsistent conditions in the present study were similar to those between the consistent, neutral, and inconsistent conditions in Navon's studies, in which his neutral stimuli maintained the hierarchical relationship between letters and thus controlled for lateral masking.

Thus, neither acuity nor lateral masking can fully account for the differences between global and local conditions in central and peripheral locations. However, the present data are consistent with Pomerantz's (1983) findings, and replicate them even when locational uncertainty is held constant. Yet, as will be shown in Experiment 2, hierarchical stimulus processing can also change when the location of the stimulus is certain, and this seems to be due to an attentional process.

Effects of letter. Somewhat surprisingly, there were large and systematic differences in RT depending on whether the target letter was an $\mathrm{H}$ or an $\mathrm{S}$ (see Figure 3). The advantage in response time observed in the globally directed condition was greater when the target was an $\mathbf{H}$ than when it was an $S$, as shown by a significant letter $x$ level interaction $[F(1,15)=18.59, p<.001]$. This effect was due in large part to the fact that the interference produced in the Inconsistent condition when subjects were locally directed was greater when the target was an $H$ than when it was an $S$. This was confirmed by a significant letter $\times$ consistency interaction $[F(2,30)=33.69$, $p<.001]$ and by a significant letter $\times$ consistency $\times$ level interaction $[F(2,30)=8.89, p<.001]$.

It is not clear why global forms were easier to ignore when the local form was an $S$ than when it was an $H$. One obvious possibility is that the local Ss in the present experiment were more salient than the local $\mathrm{Hs}$, and so suffered less interference from competing stimuli. One way this could happen would be by some gestalt grouping or contour interaction effects that favor the $S .{ }^{2}$ This argument does not receive support from the present data because these effects would occur in both the Consistent and Inconsistent conditions, since both contain a string of either local Ss or Hs (see stimulus set in Figure 1). There is no evidence that this was the case, and, if anything, RTs were faster to local Hs than to local Ss in the 

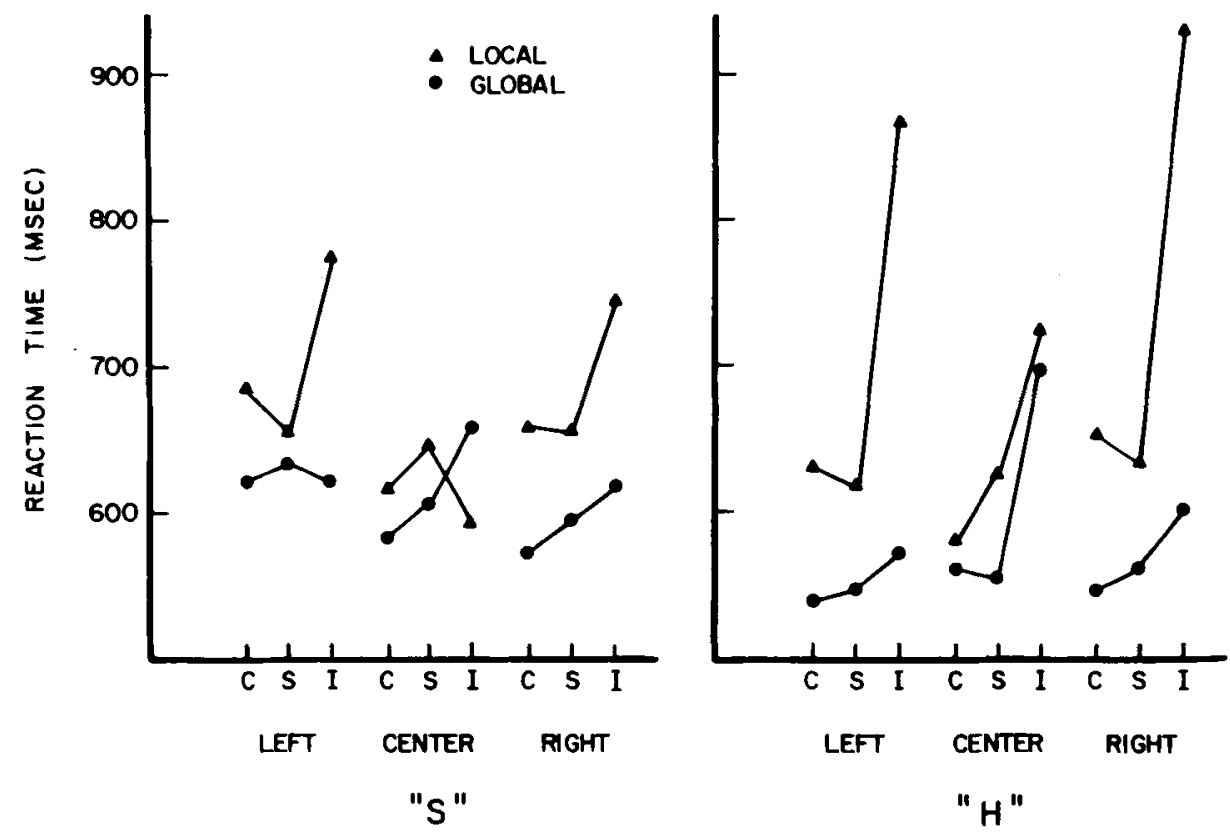

Figure 3. Reaction times for Experiment 1 for locally (triangles) and globally (circles) directed trials as a function of consistency, for stimuli presented on the left, center, or right portions of the display. (C = Consistent condition, S = Single condition, I = Inconsistent condition). Data are presented separately for the trials on which the target was an $S$ (left panel) and for those on which it was an $H$ (right panel).

Consistent condition, and the opposite was true for the Inconsistent condition. Thus, the difference in interference between the two letters cannot be attributed to such processes. Also, RT was slightly worse for local Ss than for local $\mathrm{Hs}$ in the Single condition $[F(1,15)=5.19$, $p<.05$ ], demonstrating that $S s$ are not simply easier to respond to than Hs.

Finally, the letter $\times$ location interaction also reached significance $[F(2,30)=3.58, p<.05]$ due to the fact that overall RTs were slightly faster for $\mathrm{H}$ than for $\mathrm{S}$ targets when presented to the left $[F(1,15)=5.51, p<.05]$ but not when presented in the center or to the right.

Studies of hierarchical stimulus processing have rarely included a letter factor in the data reported. Martin (1979) used a stimulus set quite similar to that used here, with stimuli composed of the letters $\mathrm{H}$ and $\mathrm{S}$, and reported only that the pattern of results "appeared to be similar" for the two letters. Neither the data nor any statistical analyses were provided. Robertson and Palmer (1983) studied mental rotation of hierarchical stimuli with patterns composed of the letters F and R. In their study, reflected local Fs were identified more quickly than reflected local Rs. These results are similar to the present data in the sense that the identity of the letter had a greater effect on local than on global processing.

\section{EXPERIMENT 2}

The results of Experiment 1 show that changes in retinal locus affect the processing of hierarchical stimuli. However, it is still possible that locational uncertainty also has some effect. The purpose of Experiment 2 was to ex- amine hierarchical stimulus processing for centrally presented stimuli in a situation where there was no locational uncertainty. The procedure was identical to that of Experiment 1, except that all stimuli were presented at the same location (i.e., in the center). In terms of an attentional hypothesis, subjects in Experiment 2 were free to attend to a smaller area of space, since all stimuli appeared in the same location, and this should result in improved performance. Experiment 2 also provided an opportunity to determine whether or not the substantial effects of letter observed in Experiment 1 would be repeated.

\section{Method}

Sixteen right-handed paid volunteers served as subjects. They ranged in age from 20 to 40 years $(M=32.5, S D=5.9)$. Eight subjects who had served in Experiment 1 also served in Experiment 2. All subjects had normal or corrected-to-normal vision. All aspects of the stimuli and procedures were identical in Experiment 1 and Experiment 2, except that all stimuli were presented centrally in Experiment 2.

\section{Results and Discussion}

As in Experiment 1, the error rate in Experiment 2 was low $(M=1.9 \%)$ and there was no indication of a speedaccuracy tradeoff between errors and RT $(r=+.15)$. RTs from Experiment 2 (along with the central data from Experiment 1) are presented in Figure 4. Overall, RTs were faster when subjects were locally directed than when they were globally directed in Experiment $2[F(1,15)=21.60$, $p<.001$ ]. The main effect of consistency was also significant $[F(2,30)=24.69, p<.001]$, resulting from the fact that RTs were slower for inconsistent than for con- 


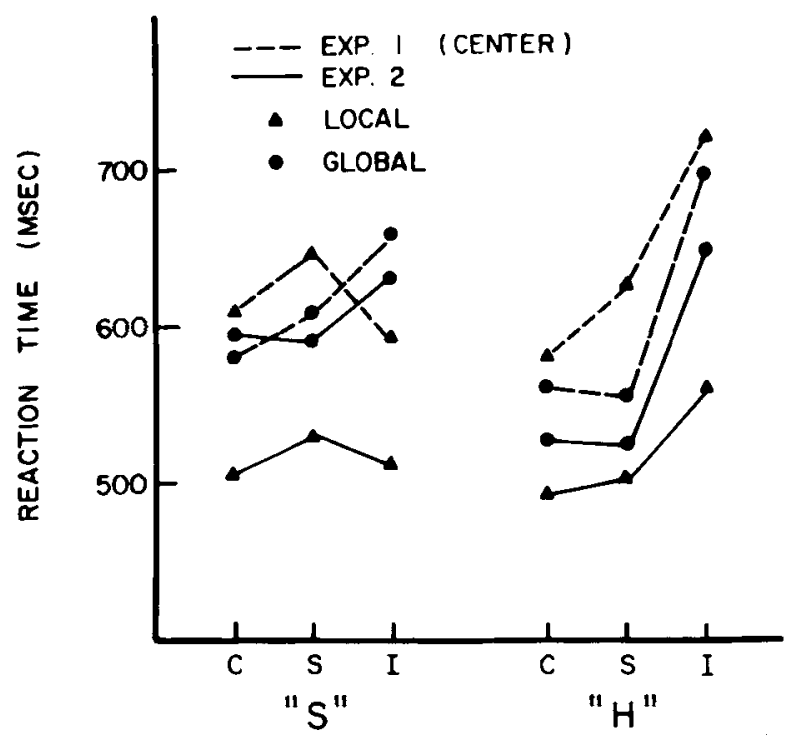

Figure 4. Reaction times for Experiment 2 (solid lines) and for the central trials of Experiment 1 (dashed lines). Data are shown for locally (triangles) and globally (circles) directed trials as a function of consistency, for stimuli presented on the left, center, or right portions of the display. ( $C$ = Consistent condition, $S=$ Single condition, I = Inconsistent condition.) Data are presented separately for the trials on which the target was an $S$ (left panel) and for those on which it was an $H$ (right panel).

sistent $[F(1,15)=29.26, p<.001]$ or single $[F(1,15)$ $=29.15, p<.001]$ trials. The effect of consistency was greater when subjects were globally directed, as reflected in a significant level $\times$ consistency interaction $[F(2,30)$ $=7.18, p<.01]$.

The results from Experiments 1 and 2 differ in that RTs were faster when subjects were locally directed in Experiment 2 but, if anything, faster when subjects were globally directed in Experiment 1. Furthermore, in Experiment 1 interference was greater when subjects were directed locally, whereas in Experiment 2 interference was greater when subjects were directed globally. These data are consistent with studies showing (1) that the relative speed of responding to local and global levels varies as a function of task demands, and (2) that the level processed more quickly will interfere more with the other level (Hoffman, 1980; Martin, 1979). As will be discussed below, however, the latter relationship does not always hold.

As was the case in Experiment 1, letter had a large effect on the pattern of results in Experiment 2. This was confirmed by a significant letter $\times$ consistency interaction $[F(2,30)=14.29, p<.001]$. This interaction arose from the fact that the main effect of consistency observed when the target was an $\mathrm{H}[F(2,30)=29.50, p<.001]$ did not occur when the target was an $S$. This was so even though there was a large advantage for the locally directed condition. In fact, the difference in mean RT between the locally and globally directed conditions was larger for S than for $\mathrm{H}$ targets. Thus, the consistency effect was smaller when the advantage in speed of processing was, if anything, larger. This finding demonstrates that the local level can enjoy a large advantage in speed of processing without interfering to any great extent with global processing. It is not clear whether this effect is restricted to local processing or whether conditions might be found in which an advantage in speed of processing at the global level might occur without the occurrence of interference at the local level.

Combined analysis. Recall that the only difference between Experiments 1 and 2 was that peripheral stimulus presentations occurred in the former but not in the latter. The central stimulus presentations were identical in the two experiments. In an attempt to evaluate differences in performance between the two experiments, an ANOVA design was conducted which compared the central data from Experiment 1 with the data from Experiment 2.

Overall, RTs were faster in Experiment 2 than in Experiment $1[F(1,30)=6.26, p<.05]$. It might be argued that part of this advantage was due to practice effects, since half of the subjects in Experiment 2 had participated first in Experiment 1. This was not the case, however, because a comparison of the performance of naive and experienced subjects in Experiment 2 produced no significant effects involving these two populations. In fact, mean RTs for naive and experienced subjects were nearly the same (554 and $550 \mathrm{msec}$, respectively).

Most of the improvement observed in Experiment $2 \alpha c$ curred in the locally directed condition. The experiment $x$ level interaction was significant $[F(1,30)=8.65$, $p<.01$ ], reflecting the fact that RTs in the locally directed condition were faster in Experiment 1 than in Experiment $2[F(1,30)=9.54, p<.01]$, whereas RTs in the globally directed condition did not differ for the two experiments. Furthermore, RTs were faster in the locally directed than in the globally directed condition in Experiment $2[F(1,15)=21.60, p<.001]$, whereas level had no significant effect for centrally presented stimuli in Experiment 1.

The improvement in performance observed in the locally directed condition in Experiment 2, as compared with the central data of Experiment 1, was uniform across the consistency conditions. That is, the amount of improvement did not differ for the Single, Inconsistent, and Consistent conditions. In other words, locational certainty increased the speed with which small letters were identified, regardless of whether those letters appeared within a hierarchy or not. Thus, locational certainty had its effect on small as opposed to local stimuli. This is in contrast to the effects produced by changes in retinal locus. As was shown in Experiment 1, such changes affected the processing of local as opposed to small stimuli.

There was no indication that the effect of consistency differed in Experiments 1 and 2, regardless of which letter served as the target. The consistency $\times$ experiment interaction was not significant, nor was any higher order interaction involving these factors. In fact, as can be seen in Figure 4, the functions relating consistency to RT are 
quite parallel for the two experiments. Responding at the local level when the target was an $\mathrm{S}$ was faster in Experiment 2 than in Experiment 1, but the effect of consistency was very similar for the two experiments. The same was true when the target was an $\mathrm{H}$. Likewise, the global consistency functions for the two experiments were parallel and this was true for both target letters.

Here, again, there was a dissociation between the relative speed of processing at the two levels and the amount of interference observed. While it is inescapable that interference cannot occur unless some information from the irrelevant level has been processed before a decision is made about the relevant level, the present data show that a large change in speed of processing can occur without any corresponding change in the amount of interference observed.

The difference in performance observed between the centrally presented stimuli in Experiments 1 and 2 cannot be accounted for by differences in acuity. RTs were faster in Experiment 2 than in Experiment 1 even though the centrally presented stimuli in the two experiments were the same in terms of visual parameters known to influence speed of processing, such as lateral masking, visual angle, or spatial frequency.

Experiments 1 and 2 differed in that there was locational uncertainty in the former but not the latter. It was suggested above that the uncertainty in Experiment 1 may have induced subjects to attend to a larger area, encompassing all three potential locations. In contrast, subjects could attend to a much smaller area in Experiment 2 because all stimuli appeared in the center. Since there is reason to believe that processing efficiency increases with decreases in the size of the attended area (Eriksen \& St. James, 1986; Eriksen \& Yeh, 1985), one would expect improved performance in Experiment 2. However, an alternative explanation is that the appearance of peripheral stimuli in Experiment 1 induced subjects to disregard the fixation instructions. If so, RTs might be slower for centrally presented stimuli in Experiment 1 because subjects moved their eyes toward the peripheral locations on some trials. Experiment 3 was conducted to determine what role, if any, eye movements played in the outcome of Experiment 1.

\section{EXPERIMENT 3}

Experiment 3 repeated the procedure used in Experiment 1 with two modifications. First, stimuli were not presented until it was determined, by the use of an eyemovement monitor, that the subject was looking at the fixation point. This procedure ensured that all stimuli impinged on the intended retinal loci, and made it possible to determine unequivocally that eye movements made no contribution to the pattern of results. Second, the mask was eliminated from the procedure in Experiment 3. The mask was included in Experiments 1 and 2 in an attempt to equate our procedure in potentially relevant respects with those used by others (e.g., Martin, 1979; Navon,
1977). However, there is reason to believe that a mask such as that used in the present experiment might be more effective in the peripheral retina than in the fovea (see Breitmeyer, 1984, for a review). Thus, it is possible that the difference between central and peripheral presentation in Experiment 1 was due to the presence of a mask rather than to retinal locus per se. The mask was eliminated in Experiment 3 to determine if this was the case.

\section{Method}

Sixteen right-handed paid volunteers served as subjects. They ranged in age from 21 to 39 years $(M=31.8, S D=5.7)$. Three subjects who had served in Experiments 1 and 2 also served in Experiment 3. All subjects had normal or corrected-to-normal vision.

The stimuli and procedures used in Experiment 3 were identical to those used in Experiment 1 with two exceptions. First, the mask was eliminated from the procedure in Experiment 3. Second, eye movements were monitored using an Applied Science Laboratories Model 210 eye-movement monitor in conjunction with a Model 115 head restraint. The eye-movement monitor was interfaced with the IBM-XT controlling the experimental events, and information about eye position was used to determine trial events. Eye position was sampled $400 \mathrm{msec}$ after onset of the fixation point and every $100 \mathrm{msec}$ thereafter. On any given trial, the fixation point stayed on until eye position was within $.5^{\circ}$ of the fixation point for two consecutive samples. As in Experiment 1, presentation of the hierarchical stimulus occurred immediately upon termination of the fixation point.

\section{Results and Discussion}

The error rate for Experiment 3 was low $(M=1.5 \%)$ and when RT and errors were compared, there was no indication of a speed-accuracy tradeoff $(r=+.03)$. The error data were not analyzed further.

Overall, the pattern of results was quite similar to that found in Experiment 1 (see Figure 5), clearly demonstrating that neither the mask nor eye movements can account for the differences found between central and peripheral presentation in Experiment 1 or for the differences between Experiments 1 and 2 for centrally presented stimuli. RTs were faster when subjects were directed globally than when they were directed locally $[F(1,15)=29.91$, $p<.001]$. There was also a large effect of consistency $[F(2,30)=27.90, p<.001]$, RTs being slower in the Inconsistent than in the Consistent $[F(1,15)=75.59$, $p<.001]$ or Single $[F(1,15)=36.71, p<.001]$ conditions. In addition, the effect of consistency was greater when subjects were directed locally than when they were directed globally, as evidenced by a significant level $X$ consistency interaction $[F(2,30)=9.21, p<.001]$.

As in Experiment 1, the pattern of results in Experiment 3 differed as a function of the location of stimulus presentation. The main effect of location was significant $[F(2,30)=6.31, p<.01]$, reflecting the fact that responses were faster for stimuli presented centrally than for those presented to the left $[F(1,15)=15.70, p<.01]$ or to the right $[F(1,15)=6.19, p<.05]$. Location also interacted with level $[F(2,30)=21.89, p<.001]$, reflecting the fact that the significant global advantage observed in the periphery $[F(1,15)=69.95, p<.001$, for 

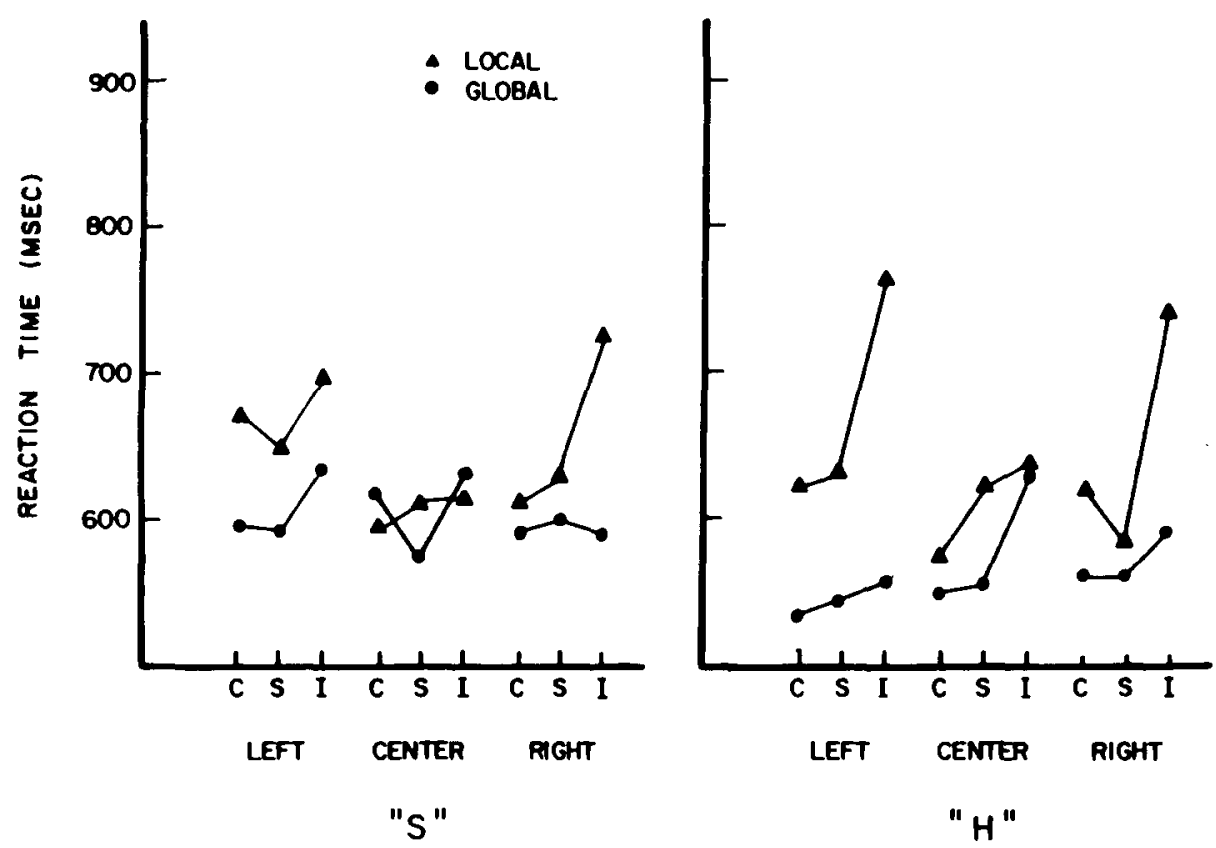

Figure 5. Reaction times for Experiment 3 for locally (triangles) and globally (circles) directed trials as a function of consistency, for stimuli presented on the left, center, or right portions of the display. ( $C$ = Consistent condition, $\mathbf{S}=$ Single condition, $\mathbf{I}=$ Inconsistent condition). Data are presented separately for the trials on which the target was an $S$ (lef panel) and for those on which it was an H (right panel).

the left and $F(1,15)=26.48, p<.001$, for the right] was not observed for stimuli presented centrally. Note that level had no significant effect on performance for centrally presented stimuli for either Experiment 1 or Experiment 3 (although there was a mean advantage in the globally directed condition in both). In contrast, there was a significant advantage for the locally directed condition in Experiment 2, in which all stimuli were centrally presented. Because of the fixation procedure used in Experiment 3, eye movement is not a viable explanation for this difference. However, an expanding and contracting attentional spotlight can account for the improved performance observed when peripheral stimuli were not presented.

As in Experiment 1, the level $\times$ consistency interaction was more pronounced for stimuli presented peripherally than for stimuli presented centrally, as evidenced by a significant location $\times$ level $\times$ consistency interaction $[F(4,60)=11.03, p<.001]$. Unlike in Experiment 1, the location $\times$ consistency interaction did not reach significance in Experiment 3. This seems to have resulted from there being less interference (particularly at the local level) for peripherally presented stimuli in Experiment 3 than in Experiment 1 . The reason for this difference is not known. Whatever the reason, however, it clearly cannot account entirely for the difference between peripherally and centrally presented stimuli, since most such differences remained in Experiment 3.

The data from the Single condition of Experiment 3 were quite similar to those of Experiment 1. Again, RTs in the Single condition were faster for small than for large letters $[F(1,15)=21.71, p<.001]$. However, as was the case in Experiment 1, location affected the processing of small letters only if those letters occurred in the context of a hierarchical stimulus. There was no improvement for centrally, as opposed to peripherally, presented single letters in the locally directed condition. In contrast, RTs for the locally directed condition were shorter with central than with peripheral presentation in the Consistent condition $[F(1,15)=34.55, p<.001$, for the left and $F(1,15)=9.47, p<.01$, for the right] and in the Inconsistent condition $[F(1,15)=52.57, p<.001$, for the left and $F(1,15)=14.86, p<.01$, for the right]. This pattern of results also resulted in a significant consistency $\times$ location $\times$ level interaction $[F(4,60)=11.03$, $p<.001]$. Thus, as in Experiment 1, central presentation increased the speed with which small letters could be identified but only when those letters occurred within a hierarchical stimulus.

Effects of letter. Letter effects similar to those observed in Experiment 1 were also found in Experiment 3. RTs were faster when the target was an $H$ than when it was an $S[F(1,15)=6.12, p<.05]$. In addition, the advantage in RT observed in the globally directed condition was greater when the target was an $\mathbf{H}$ than when it was an $S$, as shown by a significant letter $\times$ level interaction $[F(1,15)=6.68, p<.05]$. As in Experiment 1 , this effect was due mostly to the fact that the interference produced in the Inconsistent condition when subjects were locally directed was greater when the target was an $\mathrm{H}$ than 
when it was an $\mathrm{S}$. This was confirmed by a significant letter $\times$ consistency interaction $[F(2,30)=5.01$, $p<.01]$. Unlike in Experiment 1, the letter $\times$ consistency $\times$ level interaction was not significant in Experiment 3. However, the letter $\times$ consistency $\times$ level $\times$ location interaction was significant $[F(4,60)=5.42$, $p<.001]$.

Combined analysis. The ANOVAs conducted on the data from Experiment 3 revealed the same set of significant effects as that seen in Experiment 1 with no important exceptions. Thus, it is quite clear that the overall pattern of results was the same in Experiments 1 and 3. There were, however, differences in the magnitude of those effects between the two experiments. An ANOVA that included experiment ( 1 vs. 3 ) as a between-subjects factor revealed that subjects in Experiment 1 exhibited a somewhat larger effect of consistency than did subjects in Experiment 3 . This was revealed by a significant experiment $\times$ consistency interaction $[F(2,60)=4.75, p<.05]$. This tendency toward a larger consistency effect in Experiment 1 was more pronounced when the target was an $H$ than when it was an $S$, as shown by the significant experiment $\times$ consistency $\times$ letter interaction $[F(2,60)=$ $7.35, p<.01]$. Finally, the experiment $\times$ level $\times$ location interaction was also significant $[F(2,60)=3.79$, $p<.05]$. This reflected the fact that, even though the global advantage was larger in the periphery than in the center for both Experiments 1 and 3, this difference was larger in Experiment 1. All three of these interactions resulted from there being more of a consistency effect, especially when the target was an $H$, in Experiment 1 than in Experiment 3. Thus, the absence of a mask seems to have slightly decreased the amount of interference observed in the inconsistent condition. Again, however, it is clear that the pattern of results for the two experiments is the same.

\section{GENERAL DISCUSSION}

In Experiments 1 and 3, performance was compared for central and peripheral stimulus presentations. Central presentation resulted in faster reaction times and less interference at the local level, relative to peripheral presentation. Since both central and peripheral presentations $o c-$ curred in the context of locational uncertainty, this factor cannot account for the difference in performance observed here. In addition, Experiment 3 ruled out the possibility that these effects were related to eye movements or to the use of a mask. These data are consistent with the claim that local processing benefits more than global processing from an increase in acuity (Grice, et al., 1983; Pomerantz, 1983). However, central presentation benefited the processing of local letters as opposed to letters of a certain size (i.e., small letters). Yet acuity might be expected to affect small letters regardless of the context in which they occur, and it was true, in both experiments, that small letters were identified faster in the center than in the periphery if they appeared in one of the hierarchical patterns but not if they appeared alone. Of course, spatial interactions do occur (e.g., lateral masking), but these effects cannot account for the differences between consistent and inconsistent conditions, or for the fact that stimuli that maintain the hierarchical order prouduce the same differences between global and local as the single stimuli used here (Navon, 1977). Although we cannot precisely specify the reason for differences in speed of processing between central and peripheral presentation, we can conclude that locational uncertainty was not the source of these differences.

A more important finding occurred in Experiment 2, in which all stimuli were presented centrally so that there was no locational uncertainty. This resulted in faster responding in the locally directed condition relative to the central data of Experiment 1. Experiments 1 and 2 differed only in that the former included peripheral stimulus presentations and thus involved locational uncertainty. It has already been suggested that the uncertainty in Experiment 1 may have induced subjects to attend to a larger area, encompassing all three potential locations. In contrast, subjects could attend to a much smaller area in Experiment 2 because all stimuli appeared in the center. Since processing efficiency seems to increase with decreases in the size of the attended area, one would expect this improved performance in Experiment 2. However, the difference in performance between the two experiments was largely restricted to the local level. Global RTs did not differ, and it is not clear why such a change in attention would benefit processing only at the local level. This finding is interesting in light of studies showing that advance information as to the level at which a target will appear (as opposed to its spatial location) affects processing at the local and global levels symmetrically (Kinchla, Solis-Macias, \& Hoffman, 1983; Lamb \& Robertson, 1987; Robertson, Lamb, \& Knight, in press). This suggests the possibility that the relative speed with which local and global levels are processed might be modulated by two separate attentional mechanismsone that selects information by hierarchical level and one that selects information by spatial location and extent.

One of the more surprising aspects of the present set of data was the large size of the effects produced by the different target letters. In all the experiments reported here, subjects could more easily ignore the global form when the local form was an $S$ than when it was an $\mathrm{H}$. The reason for this is not obvious, and because the effects of letter are so rarely reported, it is not certain how common this finding might be. Clearly, however, stimulus sets should be chosen with care, and this factor should be included when data are analyzed if results are to be safely interpreted as relevant to hierarchical stimulus processing in general.

The analysis of letter also revealed an interesting and unusual relationship between the relative speed with which local and global levels are processed and Stroop-type interference. The comparison between the central data of Experiments 1 and 2 revealed that there was little interference when the target was an $S$ but significant mutual interference when the target was an $H$, and this was true 
for all experiments. Despite this difference in interference, the relative speed of processing did not differ as a function of letter. In other words, interference, but not relative speed of processing, varied as a function of letter. In contrast, there was a large local advantage in Experiment 2 but, if anything, a global advantage in Experiment 1 , and this was true for both target letters. Despite this difference in speed of responding, there was no indication that interference effects differed between the two experiments. Thus, speed of processing, but not interference, varied between the two experiments. This dissociation provides evidence that speed of processing and interference can vary independently, and suggests the possibility that the two effects arise as the result of the operation of separate mechanisms. The same general pattern emerges when the central data from Experiment 3 are considered (although the data from Experiment 3 were not directly compared with the data from Experiment 2 because of the difference in fixation and masking procedures used in the two experiments).

Other recent studies have also shown that speed of processing and interference do not always covary (Humphreys, Riddoch, \& Quilan, 1986; Lamb, Robertson, \& Knight, in press; Navon \& Norman, 1983). For example, Lamb et al., using a task very similar to that employed here, found that patients with lesions centered in temporal regions showed no interference effects, whereas patients with lesions centered in parietal regions showed normal interference. This was so even though the two groups did not differ in terms of the relative speed with which local and global letters were identified. In addition, patients with lesions in the left hemisphere showed an increased global advantage relative to controls, whereas patients with lesions in the right hemisphere did not. This was the case even though there was no indication that these groups differed in terms of interference effects. These results, along with the present data, suggest that the relative speed with which global and local letters are processed and the amount of interference observed are determined by separate mechanisms.

\section{REFERENCES}

Boer, L. C., \&euss, P. J. G. (1982). Global precedence as a postperceptual effect: An analysis of speed-accuracy tradeoff functions. Perception \& Psychophysics, 31, 358-366.

BREITMEYER, B. G. (1984). Visual masking: An integrative approach. New York: Oxford University Press.

ERIKSEN, C. W., \& S. JAMES, J. D. (1986). Visual attention within and around the field of focal attention: A zoom lens model. Perception \& Psychophysics, 40, 225-240.

ERIKSEN, C. W., \& YEH, Y. (1985). Allocation of attention in the visual field. Journal of Experimental Psychology: Human Perception \& Performance, 11, 583-597.

Grice, G. C., Canham, L., Boroughs, J. M. (1983). Forest before trees? It depends where you look. Perception \& Psychophysics, 33, 121-128.

Hofrman, J. (1980). Interaction between global and local levels of form. Journal of Experimental Psychology: Human Perception \& Performance, 6, 222-234.

Humphreys, G. W., Riddoch, J., \&uilan, T. T. (1986). Interactive processes in perceptual organization: Evidence from visual ag- nosia. In M. I. Posner \& O. S. M. Marin (Eds.), Attention and performance $X I$ (pp. 301-318). Hillsdale, NJ: Erlbaum.

Johnston, W. A., DARK, V. J. (1986). Selective attention. In M. R. Rosenzwieg \& L. W. Porter (Eds.), Annual review of psychology (p. 43-75). Palo Alto, CA: Annual Reviews.

JONIDES, J. (1983). Further toward a model of the mind's eye. Bulletin of the Psychonomic Society, 21, 247-250.

KImchı, R., PAl.mer, S. E. (1982). Form and texture in hierarchically constructed patterns. Joumal of Experimenial Psychology: $\mathrm{Hu}$ man Perception \& Peformance, 8, 521-535.

Kinchla, R. A., Solis-Macias, V., Hoffman, I. (1983). Attending to different levels of structure in a visual image. Perception \& Psychophysics, 33, 1-10.

KINCHLA, R. A., WOLFE, J. (1979). The order of visual processing: "Top down," "bottom up," or "middle out." Perception \& Psychophysics, 25, 225-231.

KoffKA, K. (1935). Principles of Gestalt psychology. New York: Academic Press.

LAmb, M. R., Robertson, L. C. (1987). The effects of acute alcohol on attention and the processing of hierarchical patterns. Alcoholism: Clinical \& Experimental Research, 11, 243-248.

LAmb, M. R., Robertson, L. C., KNIGHT, R. T. (in press). Attention and interference in the processing of global and local information: Effects of unilateral temporal-parietal junction lesions. Neuropsychologia.

MARTIN, M. (1979). Local and global processing: The role of sparsity. Memory \& Cognition, 7, 476-484.

MiLLER, J. (1981). Global precedence in attention and decision. Journal of Experimental Psychology: Human Perception \& Performance, 7, 1161-1174.

Navon, D. (1977). Forest before trees: The precedence of global features in visual perception. Cognitive Psycholoogy, 9, 353-383.

NAvon, D. (1981). Do attention and decision follow perception? Comment on Miller. Journal of Experimental Psychology: Human Perception \& Performance, 7, 1175-1182.

Navon, D., Norman, J. (1983). Does global precedence really depend on visual angle? Journal of Experimental Psychology: Human Perception \& Performance, 9, 955-965.

Pomerantz, J. R. (1983). Global and local precedence: Selective attention in form and motion perception. Journal of Experimental Psychology: General, 112, 516-540.

RoberTson, L. C. (1986). From gestalt to neo-gestalt. In T. J. Knapp \& L. C. Robertson (Eds.), Aproaches to recognition: Contrasts and controversies (pp. 159-188). Hillsdale, NJ: Erlbaum.

Robertson, L. C., LAMB, M. R., KNight, R. T. (in press). Effects of lesions of temporal-parietal junction on perceptual and attentional processing in humans. Joumal of Neuroscience.

Robertson, L. C., PAlmer, S. E. (1983). Holistic processes in the perception and transformation of disoriented figures. Journal of $E x$ perimental Psychology: Human Perception \& Performance, 9, 203-214.

STROOP, J. R. (1935). Studies of interference in serial verbal reactions. Journal of Experimental Psycholoogy, 18, 643-661.

TrrCHNER, E. (1909). Experimental psychology of the thought processes. New York: MacMillan.

WARD, L. M. (1982). Determinants of attention to local and global features of visual forms. Journal of Experimental Psychology: Human Perception \& Performance, 8, 562-581.

\section{NOTES}

1. Although Navon found no difference in RT between single large and small letters, he did not present these stimuli peripherally. Thus, we could not compare our single-letter conditions with his. This is not critical for the lateral masking issue, since we could compare our single conditions to his neutral conditions, in which the stimuli were presented peripherally.

2. This possibility was suggested by an anonymous reviewer.

(Manuscript received May 18, 1987; revision accepted for publication January 5,1988 .) 